\title{
On generalized García-Falset coefficient in Musielak-Orlicz sequence spaces
}

Haifeng Ma*

\section{"Correspondence:}

haifengma@aliyun.com

School of Mathematical Science,

Harbin Normal University, Harbin,

150025, China

\begin{abstract}
We introduce a new geometric coefficient which is related García-Falset coefficient and weak star fixed point property. The García-Falset coefficient that was introduced by Benavides in (Houst. J. Math. 22:835-849, 1996) is calculated in this paper for Musielak-Orlicz sequence spaces equipped with the Luxemburg norm. Specifically, in reflexive Banach spaces, the new geometric coefficient and the García-Falset coefficient are the same.
\end{abstract}

MSC: 46B20; 46E30; 47H09

Keywords: generality García-Falset coefficient; weak fixed point property; Musielak-Orlicz sequence spaces

\section{Introduction and preliminaries}

Throughout this paper $X$ is a Banach space which is assumed not to have the Schur property, i.e., $X$ has a weakly convergent sequence that is not norm convergent. $S(X)$ and $B(X)$ denote the unit sphere and the unit ball of $X$, respectively and $l^{0}$ denotes the set of all real sequences.

A Banach space $X$ is said to have the fixed point property (FPP, for short) if every nonexpansive mapping $T: C \rightarrow C$, i.e., the mapping satisfying

$$
\|T x-T y\| \leq\|x-y\|, \quad \forall x, y \in C,
$$

and acting on a nonempty bounded closed and convex subset $C$ of $X$ has a fixed point in $C$. A natural generalization of FPP is the weak fixed point property (WFPP, for short). A Banach space $X$ is said to have the WFPP whenever it satisfies the above condition from the definition of FPP with 'weakly compact' in place of 'bounded closed'. In 1965 Kirk [2] proved that any reflexive Banach space with normal structure has the FPP. In 1989 Prus [3] introduced a property of a Banach space $X$, called nearly uniformly smoothness. In 1992 Prus [4] also proved that a weakly nearly uniformly smooth Banach space $X$ with the weak Opial property has the FPP. To obtain the weak fixed point property in Banach spaces, García-Falset introduced in [5] the following coefficient:

$$
R(X)=\sup \left\{\liminf _{n \rightarrow \infty}\left\|x_{n}-x\right\|:\left\{x_{n}\right\} \subset B(X), x_{n} \stackrel{w}{\rightarrow} 0, x \in B(X)\right\} .
$$

(c) $2015 \mathrm{Ma}$. This article is distributed under the terms of the Creative Commons Attribution 4.0 International License (http://creativecommons.org/licenses/by/4.0/), which permits unrestricted use, distribution, and reproduction in any medium, provided you give appropriate credit to the original author(s) and the source, provide a link to the Creative Commons license, and indicate if changes were made. 
He has proved that a Banach space $X$ with $R(X)<2$ has the weak fixed point property, i.e., every nonexpansive mapping $T$ from a weakly compact nonempty and convex set $A \subset X$ into itself has a fixed point in $A$ (see [6] and [7]).

A Banach space $X$ is said to be NUS provided that for every $\varepsilon>0$ there is $\eta>0$ such that if $t \in(0, \eta)$ and $\left\{x_{n}\right\}$ is a basic sequence in $B(X)$, then there exists $k>1$ so that $\left\|x_{1}+t x_{k}\right\| \leq$ $1+t \varepsilon$ (see [3]).

A natural generalization of this notion is said to be WNUS. A Banach space $X$ is WNUS whenever it satisfies the above condition with 'for some $\varepsilon \in(0,1)$ ' in place of 'for every $\varepsilon>0$ ' (see [6]).

It is well known that a Banach space $X$ is WNUS if and only if $X$ is reflexive and $R(X)<2$ (see [6]).

The coefficient $R(X, a)$ of a Banach space $X$ was defined in 1996 by Benavides [1], as a generalization of the coefficient $R(X)$, which also plays an important role in the fixed point theory for nonexpansive mappings. Benavides defined the coefficient $R(X, a)$ for a Banach space $X$ as follows.

Definition 1.1 For a given $a \geq 0$,

$$
R(X, a)=\sup \left\{\liminf _{n \rightarrow \infty}\left\|x_{n}-x\right\|: x_{n} \in B(X), x_{n} \stackrel{w}{\rightarrow} 0, D\left[\left(x_{n}\right)\right] \leq 1,\|x\| \leq a\right\},
$$

where $D\left[\left(x_{n}\right)\right]=\liminf _{n \rightarrow \infty}\left\{\left\|x_{i}-x_{j}\right\|: i \neq j, i, j \geq n\right\}$.

He also defined the following coefficient:

$$
M(X)=\sup \left\{\frac{1+a}{R(X, a)}: a \geq 0\right\}
$$

Moreover, the coefficient $R(X, a)$ remains unaltered if in the definition we replace liminf by $\lim$ sup. He obtained the following: for a given $a \geq 0$, if $R(X, a)<1+a$, then the Banach space $X$ has the fixed point property; the result means that the condition $M(X)>1$ implies that $X$ has the weak fixed point property for nonexpansive mappings [1].

In this paper, we introduce a new geometric coefficient that is a related García-Falset coefficient and a weak* fixed point property, written $R^{*}\left(X^{*}, a\right)$. For a given $a \geq 0$, let

$$
R^{*}\left(X^{*}, a\right)=\sup \left\{\lim _{n \rightarrow \infty} \inf \left\|x_{n}-x\right\|: x_{n} \in B\left(X^{*}\right), x_{n} \stackrel{w^{*}}{\rightarrow} 0, D\left[\left(x_{n}\right)\right] \leq 1,\|x\| \leq a, x \in X\right\},
$$

where $D\left[\left(x_{n}\right)\right]=\lim _{n \rightarrow \infty} \inf \left\{\left\|x_{i}-x_{j}\right\|: i \neq j, i, j \geq n\right\}$.

Similarly to [1], we can prove that if $R^{*}\left(X^{*}, a\right)<1+a$ for some $a \in(0,1]$ then $T: C \rightarrow C$ has a fixed points $a \in C$, if $C$ is a weak*-compact and weak* sequentially complete subset of $X^{*}$ and $T$ is a nonexpansive mapping. It is clear that $R(X, a)=R^{*}\left(X^{*}, a\right)$ if the Banach space $X$ is reflexive.

A Banach space $X$ is called a Köthe sequence space if it is a subspace of $l^{0}$ and for every $x \in l^{0}$ and $y \in X$ satisfying $|x(i)| \leq|y(i)|$ for all $i \in \mathcal{N}$, we have $x \in X$ and $\|x\| \leq\|y\|$ and if there is a $x=(x(i)) \in X$ with $x(i)>0$ for all $i \in \mathcal{N}$ (see [8,9] and [10]).

Let

$$
X_{a}=\left\{x \in X: \lim _{n \rightarrow \infty}\|(0,0, \ldots, 0, x(n+1), x(n+2), \ldots)\|=0\right\} .
$$


A Köthe sequence space $X$ is said to have an absolutely continuous norm if $X_{a}=X$. A Köthe sequence space $X$ is said to have the semi-Fatou property if for every sequence $\left\{x_{n}\right\} \subset X$ and $x \in X$ satisfying $\left|x_{n}(i)\right| \uparrow|x(i)|$ for all $i \in N$ we have $\left\|x_{n}\right\| \rightarrow\|x\|$.

A mapping $\Phi: \mathcal{R} \rightarrow \mathcal{R}_{+}$is said to be an Orlicz function if $\Phi$ vanishes only at $0, \Phi$ is even and convex on the whole line $R$. For every Orlicz function $\Phi$ we define its complementary function $\Psi: \mathcal{R} \rightarrow[0, \infty)$ by the formula

$$
\Psi(v)=\sup _{u>0}\{u|v|-\Phi(u)\}
$$

for every $v \in \mathcal{R}$.

Denote by $\Phi=\left\{\Phi_{i}\right\}_{i=1}^{\infty}$ a sequence Orlicz function. Such a sequence is called a MusielakOrlicz function on $N \times R$.

We say that $\Phi$ satisfies the $\delta_{2}$-condition ( $\Phi \in \delta_{2}$ for short) if there exist $k>0, u_{0}>0$,and $c_{i} \geq 0$, with $\sum_{i \geq 1} c_{i}<\infty$ such that we have the inequality

$$
\Phi_{i}(2 u) \leq k \Phi_{i}(u)+c_{i} \quad\left(i \geq 1, \Phi_{i}(u) \leq u_{0}\right)
$$

We now introduce a new definition, namely $\bar{\delta}_{2}(k)$. We say that $\Phi$ satisfies the $\bar{\delta}_{2}(k)$ condition $\left(\Phi \in \bar{\delta}_{2}(k)\right.$ for short) if there exist $\varepsilon \in(0,1)$ and $i_{\varepsilon} \in \mathcal{N}$ such that

$$
I_{\Phi}\left(\frac{x}{2}\right) \leq \frac{1-\varepsilon}{2} I_{\Phi}(x)
$$

whenever $I_{\Phi}(x)=k$ and $N(x) \geq i_{\varepsilon}$, where $N(x)=\{i \in \mathcal{N}: x(i) \neq 0\}$ and $N(x) \geq i_{\varepsilon}$, which means that $\min \{i: i \in N(x)\} \geq i_{\varepsilon}$.

Proposition 1.2 The following are equivalent (see [11]):

(1) $\Phi \in \delta_{2}$;

(2) for any $\varepsilon>0$, there exist $k_{\varepsilon}>0, u_{\varepsilon}>0$, and $c_{i} \geq 0(i \geq 1), \sum_{i \geq 1} c_{i}<\infty$ such that

$$
\Phi_{i}\left(\frac{u}{\varepsilon}\right) \leq k_{\varepsilon} \Phi_{i}(u)+c_{i} \quad\left(i \geq 1, \Phi_{i}(u) \leq u_{\varepsilon}\right)
$$

(3) there exist $\varepsilon \in(0,1), i_{\varepsilon} \in M, c_{i} \geq 0(i \geq 1), \sum_{i \geq 1} c_{i}<\infty$ and $v_{\varepsilon}>0$ such that

$$
\Psi_{i}\left(\frac{v}{2}\right) \leq \frac{1-\varepsilon}{2} \Psi_{i}(v)+c_{i} \quad\left(i \geq 1, \Psi_{i}(v) \leq v_{\varepsilon}\right)
$$

The Musielak-Orlicz sequence space $l_{\Phi}$ is defined to be the set $\left\{x \in l_{0}: I_{\Phi}(\lambda x)=\right.$ $\sum_{i=1}^{\infty} \Phi_{i}(\lambda x(i))<\infty$ for some $\left.\lambda>0\right\}$ and its subspace $h_{\Phi}$ is defined to be the set $\left\{x \in l_{0}\right.$ : $I_{\Phi}(\lambda x)=\sum_{i=1}^{\infty} \Phi_{i}(\lambda x(i))<\infty$ for any $\left.\lambda>0\right\}$ both equipped with the Luxemburg norm

$$
\|x\|=\inf \left\{k>0: I_{\Phi}\left(\frac{x}{k}\right) \leq 1\right\} .
$$

To simplify notations, we put $l_{\Phi}=\left(l_{\Phi},\|\|_{\Phi}\right)$ and $h_{\Phi}=\left(h_{\Phi},\|\|_{\Phi}\right)$.

We say that a Musielak-Orlicz function $\Phi$ satisfies the $\bar{\delta}_{2}$-condition if its complementary function $\Psi$ satisfies the $\delta_{2}$-condition.

The basic information on Musielak-Orlicz spaces can be found in [11-13], and [14]. 


\section{Results}

The idea of Theorem 2.1 is similar to Corollary 8.2 in [15]. In order to keep the consistency of this paper, we accept it in the following.

Theorem 2.1 Let X be a Köthe sequence space with the Fatou property. If X has no absolute continuous norm, then $R(X, a)=1+a$.

Proof Suppose that $X$ does not have an absolutely continuous norm. Then there exists $\varepsilon_{0}>0$ and $x_{0} \in S(X)$ such that

$$
\lim _{n \rightarrow \infty}\left\|\sum_{i=n+1}^{\infty} x_{0}(i) e_{i}\right\|=\varepsilon_{0},
$$

where $e_{i}=(0,0, \ldots, 1,1,0, \ldots)$.

Take a sequence of positive numbers $\left\{\varepsilon_{n}\right\}$ such that $\varepsilon_{n} \downarrow 0$. By $\lim _{n \rightarrow \infty}\left\|\sum_{i=n+1}^{\infty} x_{0}(i) e_{i}\right\|=$ $\varepsilon_{0}$, there exists $n_{1} \in N$ such that

$$
\left\|\sum_{i=n_{1}}^{\infty} x_{0}(i) e_{i}\right\| \leq\left(1+\varepsilon_{1}\right) \varepsilon_{0}
$$

Notice that

$$
\lim _{m \rightarrow \infty}\left\|\sum_{i=n_{1}+1}^{m} x_{0}(i) e_{i}\right\|=\varepsilon_{0},
$$

so there exists $n_{2}>n_{1}$ such that

$$
\left(1-\varepsilon_{1}\right) \varepsilon_{0} \leq\left\|\sum_{i=n_{1}+1}^{n_{2}} x_{0}(i) e_{i}\right\| \leq\left(1+\varepsilon_{1}\right) \varepsilon_{0} .
$$

In this way, we get by induction a sequence $\left\{n_{i}\right\}$ of natural numbers such that

$$
\left(1-\varepsilon_{i}\right) \varepsilon_{0} \leq\left\|\sum_{j=n_{i}+1}^{n_{i+1}} x_{0}(i) e_{i}\right\| \leq\left(1+\varepsilon_{i}\right) \varepsilon_{0}, \quad i=1,2, \ldots
$$

Put $x_{i}=\sum_{j=n_{i}+1}^{n_{i+1}} x_{0}(i) e_{i}$ and $v_{k}=\sum_{j=n_{k}+1}^{\infty} x_{0}(i) e_{i}$. Then:

(a) $\left\|x_{i}\right\| \rightarrow \varepsilon_{0}$ as $i \rightarrow \infty$.

(b) $x_{i} \stackrel{w}{\rightarrow} 0$ as $i \rightarrow \infty$. It is well known that for any Köthe space $X$ we have

$$
X^{*}=X^{\prime} \oplus S
$$

where $S$ is the space of all singular functionals over $X$, i.e., functionals which vanish on the subspace $X_{a}=\{x \in X: x$ has absolutely continuous norm $\}$ and $X^{\prime}=\left\{y \in l^{0}: \sum_{i=1}^{\infty} x(i) y(i)<\right.$ $\infty$ for all $x \in X\}$ (see [11]). This means that every $f \in X^{*}$ is uniquely represented in the form

$$
f=T_{y}+\varphi
$$


where $\varphi \in S$ and for $y \in X^{\prime}$ the function $T_{y}$ is defined by

$$
T_{y}(x)=\sum_{i=1}^{\infty} x(i) y(i)
$$

for all $x \in X$.

Taking any $y \in X$, we have

$$
\lim _{i \rightarrow \infty} \sum_{j=1}^{\infty} x_{n}(j) y(j)=\lim _{i \rightarrow \infty} \sum_{j=n_{i}+1}^{n_{i+1}} x_{i}(j) y(j)=0 .
$$

(c) Put $z_{i}=\frac{x_{i}}{\left\|x_{i}\right\|}$ and $w_{k}=\frac{v_{k}}{\left\|v_{k}\right\|}$ for all $i, k \in \mathcal{N}$. It is easy to check $D\left(z_{k}\right) \leq 1$. Then

$$
\begin{aligned}
& \liminf _{i \rightarrow \infty}\left\|z_{i}+a w_{k}\right\| \\
&=\liminf _{i \rightarrow \infty}\left\|\frac{x_{i}}{\left\|x_{i}\right\|}+a \frac{v_{k}}{\left\|v_{k}\right\|}\right\| \\
&=\liminf _{i \rightarrow \infty} \frac{1}{\left\|x_{i}\right\|\left\|v_{k}\right\|}\|\| v_{k}\left\|x_{i}+a\right\| x_{i}\left\|v_{k}\right\| \\
& \geq \frac{1}{\varepsilon_{0}\left(1+\varepsilon_{k}\right) \varepsilon_{0}} \liminf _{i \rightarrow \infty}\|\| v_{k}\left\|x_{i}+a\right\| x_{i}\left\|v_{k}\right\| \\
&=\frac{1}{\varepsilon_{0}\left(1+\varepsilon_{k}\right) \varepsilon_{0}} \liminf _{i \rightarrow \infty}\left\|\left(\left\|v_{k}\right\|+a\left\|x_{i}\right\|\right) x_{i}+a\right\| x_{i}\left\|\left(v_{k}-x_{i}\right)\right\| \\
& \geq \frac{1}{\varepsilon_{0}\left(1+\varepsilon_{k}\right) \varepsilon_{0}}\left(\liminf _{i \rightarrow \infty}\left(\varepsilon_{0}+a\left(1-\varepsilon_{i}\right) \varepsilon_{0}\right)\left\|x_{i}\right\|-\limsup _{i \rightarrow \infty}\left(\left\|v_{k}\right\|-\left\|x_{i}\right\|\right)\left\|x_{i}\right\|\right) \\
& \geq \frac{1}{\varepsilon_{0}\left(1+\varepsilon_{k}\right) \varepsilon_{0}}\left(\liminf _{i \rightarrow \infty}\left(\varepsilon_{0}+a\left(1-\varepsilon_{i}\right) \varepsilon_{0}\right)\left(1-\varepsilon_{i}\right) \varepsilon_{0}-\limsup _{i \rightarrow \infty}\left(\left\|v_{k}\right\|-\left\|x_{i}\right\|\right)\left(1+\varepsilon_{i}\right) \varepsilon_{0}\right) \\
& \geq \frac{1}{\varepsilon_{0}\left(1+\varepsilon_{k}\right) \varepsilon_{0}}\left(\liminf _{i \rightarrow \infty}\left(\varepsilon_{0}+a\left(1-\varepsilon_{i}\right) \varepsilon_{0}\right)\left(1-\varepsilon_{i}\right) \varepsilon_{0}\right. \\
&\left.-\limsup _{i \rightarrow \infty}\left(\left(1+\varepsilon_{k}\right) \varepsilon_{0}-\left(1-\varepsilon_{i}\right) \varepsilon_{0}\right) \varepsilon_{0}\right) \\
&= \frac{1}{\varepsilon_{0}\left(1+\varepsilon_{k}\right) \varepsilon_{0}}\left(\left(\varepsilon_{0}+a \varepsilon_{0}\right) \varepsilon_{0}-\left(\left(1+\varepsilon_{k}\right) \varepsilon_{0}-\varepsilon_{0}\right) \varepsilon_{0}\right) \\
&= \frac{1}{\varepsilon_{0}\left(1+\varepsilon_{k}\right)}\left(\left(\varepsilon_{0}+a \varepsilon_{0}\right)-\varepsilon_{k} \varepsilon_{0}\right) \\
&= \frac{1}{\left(1+\varepsilon_{k}\right)}\left((1+a)-\varepsilon_{k}\right) .
\end{aligned}
$$

By the arbitrariness of $k$ and $\lim _{k \rightarrow \infty} \varepsilon_{k}=0$, we get $R(X, a) \geq 1+a$. It is clear that $R(X, a) \leq$ $1+a$. Therefore $R(X, a)=1+a$.

Corollary 2.2 If $\Phi \notin \delta_{2}$ then $R\left(l_{\Phi}, a\right)=R^{*}\left(l_{\Phi}, a\right)=1+a$ for any $0<a \leq 1$.

Proof Since $\Phi \notin \bar{\delta}_{2}$, we see that $l_{\Phi}$ has no absolutely continuous norm. So we have $R\left(l_{\Phi}, a\right)=1+a$. Since $h_{\Psi}$ is separable and $\left(h_{\Psi}\right)^{*}=l_{\Phi}$, we have $R^{*}\left(l_{\Phi}, a\right)=1+a$. 
For any $x \in \ell_{\Phi}$ with $\|x\|=a$ and $N(x)=\{i \in N: x(i) \neq 0\}$ being finite, we define $c_{x}$ by the formula

$$
c_{x}=\lim _{n \rightarrow \infty} \sup \left\{c_{x, y}>0: I_{\Phi}\left(\frac{x}{c_{x, y}}\right)+I_{\Phi}\left(\frac{y}{c_{x, y}}\right)=1: y \in \ell_{\Phi}, I_{\Phi}(y) \leq \frac{1}{2}, n \leq N(y)<\infty\right\} .
$$

Theorem 2.3 Suppose that $\Phi \in \delta_{2}$. Then for the Musielak-Orlicz sequence space $\ell_{\Phi}$ we have

$$
R^{*}\left(\ell_{\Phi}, a\right)=\sup \left\{c_{x}: x \in \ell_{\Phi} \text { with }\|x\|=a \text { and } N(x) \text { being finite }\right\}
$$

Proof Let

$$
d_{\Phi}=\sup \left\{c_{x}: x \in \ell_{\Phi} \text { with }\|x\|=a \text { and } N(x) \text { being finite }\right\} .
$$

Then for any $\varepsilon \in\left(0, d_{\Phi}\right)$, there exists $\|x\|=a$ with finite $N(x)$ such that

$$
c_{x}>d_{\Phi}-\varepsilon
$$

By the definition of $c_{x}$ there exists $n_{1} \in \mathcal{N}$ such that

$$
\sup \left\{c_{x, y}>0: I_{\Phi}\left(\frac{x}{c_{x, y}}\right)+I_{\Phi}\left(\frac{y}{c_{x, y}}\right)=1 \text { for } I_{\Phi}(y) \leq \frac{1}{2} \text { and } N(y) \geq n_{1}\right\}>d_{\Phi}-\varepsilon
$$

whenever $n \geq n_{1}$. By the definition of the supremum, there exists $y_{1} \in S\left(\ell_{\Phi}\right)$ with $N\left(y_{1}\right)>n_{1}$ such that $c_{x, y_{1}}>d_{\Phi}-\varepsilon$, i.e., $I_{\Phi}\left(\frac{x}{d_{\Phi}-\varepsilon}\right)+I_{\Phi}\left(\frac{y_{1}}{d_{\Phi}-\varepsilon}\right)>1$. Hence there exists $n_{2}>n_{1}$ such that $I_{\Phi}\left(\frac{x}{d_{\Phi}-\varepsilon}\right)+\sum_{i=n_{1}+1}^{n_{2}} \Phi_{i}\left(\frac{y_{1}(i)}{d_{\Phi}-\varepsilon}\right)>1$. Since $n_{2}>n_{1}$, we also have

$$
\sup \left\{c_{x, y}>0: I_{\Phi}\left(\frac{x}{c_{x, y}}\right)+I_{\Phi}\left(\frac{y}{c_{x, y}}\right)=1 \text { for } I_{\Phi}(y) \leq \frac{1}{2} \text { and } N(y) \geq n_{2}\right\}>d_{\Phi}-\varepsilon
$$

There exists $y_{2} \in \ell_{\Phi}$ with $I_{\Phi}(y) \leq \frac{1}{2}$ and $N\left(y_{2}\right)>n_{1}$ such that $c_{x, y_{2}}>d_{\Phi}-\varepsilon$, i.e., $I_{\Phi}\left(\frac{x}{d_{\Phi}-\varepsilon}\right)+$ $I_{\Phi}\left(\frac{y_{2}}{d_{\Phi}-\varepsilon}\right)>1$. Hence there exists $n_{2}>n_{1}$ such that $I_{\Phi}\left(\frac{x}{d_{\Phi}-\varepsilon}\right)+\sum_{i=n_{1}+1}^{n_{2}} \Phi_{i}\left(\frac{y_{2}(i)}{d_{\Phi}-\varepsilon}\right)>1$. Furthermore, there exists $n_{3}>n_{2}$ such that $I_{\Phi}\left(\frac{x}{d_{\Phi}-\varepsilon}\right)+\sum_{i=n_{2}+1}^{n_{3}} \Phi_{i}\left(\frac{y_{2}(i)}{d_{\Phi}-\varepsilon}\right)>1$. In such a way, we can prove by induction that there exist a sequence $\left\{y_{k}\right\}_{k=1}^{\infty} \subset \ell_{\Phi}$ with $I_{\Phi}\left(y_{k}\right) \leq \frac{1}{2}$ for any natural $k$ and a sequence of natural numbers $n_{1}<n_{2}<n_{3}<\cdots$ such that $I_{\Phi}\left(\frac{x}{d_{\Phi}-\varepsilon}\right)+$ $\sum_{i=n_{k}+1}^{n_{k+1}} \Phi_{i}\left(\frac{y_{k}(i)}{d_{\Phi}-\varepsilon}\right)>1$ for all $k \in \mathcal{N}$. It is clear that $y_{k}$ is weakly star convergent to 0 . Since the supports of $y_{k}$ are pairwise disjoint, we have $I_{\Phi}\left(y_{i}-y_{i}\right)=I_{\Phi}\left(y_{i}\right)+I_{\Phi}\left(y_{i}\right) \leq \frac{1}{2}+\frac{1}{2}=1$ for $i, j \in N$ with $i \neq j$. Therefore, $D\left[\left(y_{k}\right)\right] \leq 1$.

For any $k>i_{0}$, we have

$$
I_{\Phi}\left(\frac{y_{k}-x}{d_{\Phi}-\varepsilon}\right)=\sum_{i=n_{k}+1}^{n_{k+1}} \Phi_{i}\left(\frac{y_{k}(i)}{d_{\Phi}-\varepsilon}\right)+I_{\Phi}\left(\frac{x(i)}{d_{\Phi}-\varepsilon}\right)>1
$$

i.e., $\left\|y_{k}-x\right\|>d_{\Phi}-\varepsilon$. Therefore, $R^{*}\left(\ell_{\Phi}, a\right) \geq d_{\Phi}-\varepsilon$ and by the arbitrariness of $\varepsilon>0$, we have $R^{*}\left(\ell_{\Phi}, a\right) \geq d_{\Phi}$. 
Now, we will prove that $R^{*}\left(\ell_{\Phi}, a\right) \leq d_{\Phi}$. By the definition of $d_{\Phi}$, we always have

$$
\lim _{n \rightarrow \infty} \sup \left\{c_{x, y}>0: I_{\Phi}\left(\frac{x}{c_{x, y}}\right)+I_{\Phi}\left(\frac{y}{c_{x, y}}\right)=1 \text { for } y \in S\left(\ell_{\Phi}\right) \text { and } N(y) \geq n\right\} \leq d_{\Phi}
$$

for any $\|x\|=a$ with finite $N(x)$.

First of all, we want to prove that for any weak star null sequence $\left\{x_{n}\right\} \subset l_{\Phi}$ and $\varepsilon>0$ there exists a subsequence $\left\{x_{n_{i}}\right\} \subset\left\{x_{n}\right\}$ such that $I_{\Phi}\left(x_{n_{i}}\right) \leq \frac{1}{2}+\varepsilon$ for each $i \in N$. Otherwise there exists $\varepsilon_{0}>0$. Without loss of generality, we may assume that $I_{\Phi}\left(x_{n}\right)>\frac{1}{2}+\varepsilon_{0}$ for all $n \in \dot{N}$. By $\Phi \in \delta_{2}$ there exists a $\delta_{1}>0$ such that $\|x\|>1+5 \delta_{1}$ whenever $I_{\Phi}(x)>1+\frac{2 \varepsilon_{0}}{3}$. Using $\Phi \in \delta_{2}$ again, there exists a $\delta_{2}>0$ such that $I_{\Phi}(x)<\delta_{2}$ whenever $\|x\|<\frac{\varepsilon_{0}}{3}$. Set $\delta_{0}=$ $\min \left\{\delta_{1}, \delta_{2}\right\}$.

Put $n_{1}=1$. Then there exists a $i_{1}>1$ such that $\left\|\sum_{i=i_{1}+1}^{\infty} x_{n_{1}}(i) e_{i}\right\|<\delta_{0} \leq \delta_{2}$. Since the sequence $\left\{x_{n}\right\}$ is a weakly null sequence, there exists $n_{2}>n_{1}$ such that

$$
\left\|\sum_{i=1}^{i_{1}} x_{n}(i) e_{i}\right\|<\delta_{0} \leq \delta_{2} \quad \text { whenever } n \geq n_{2}
$$

Using $\Phi \in \delta_{2}$ again, we see that there exists a $i_{2}>i_{1}$ such that $\left\|\sum_{i=i_{2}+1}^{\infty} x_{n_{2}}(i) e_{i}\right\|<\delta_{0} \leq \delta_{2}$. Hence

$$
I_{\Phi}\left(\sum_{i=1}^{i_{1}} x_{n_{2}}(i) e_{i}\right)<\frac{\varepsilon_{0}}{3} \quad \text { and } \quad I_{\Phi}\left(\sum_{i=i_{2}+1}^{\infty} x_{n_{2}}(i) e_{i}\right)<\frac{\varepsilon_{0}}{3}
$$

Therefore $I_{\Phi}\left(\sum_{i=i_{1}+1}^{i_{2}} x_{n_{2}}(i) e_{i}\right)>\frac{1}{2}+\frac{\varepsilon_{0}}{3}$.

In such a way, we get a subsequence $\left\{x_{n_{j}}\right\} \subset\left\{x_{n}\right\}$ such that

$$
I_{\Phi}\left(\sum_{i=1}^{i_{j-1}} x_{n_{j}}(i) e_{i}\right)<\frac{\varepsilon_{0}}{3}, \quad I_{\Phi}\left(\sum_{i=i_{j}+1}^{\infty} x_{n_{2}}(i) e_{i}\right)<\frac{\varepsilon_{0}}{3}
$$

and

$$
I_{\Phi}\left(\sum_{i=i_{j-1}+1}^{i_{j}} x_{n_{2}}(i) e_{i}\right)>\frac{1}{2}+\frac{\varepsilon_{0}}{3}
$$

for all $j \in N$.

So

$$
\begin{aligned}
\left\|x_{n_{k}}-x_{n_{j}}\right\|= & \| \sum_{i=1}^{i_{k-1}} x_{n_{k}}(i) e_{i}+\sum_{i=i_{k-1}+1}^{i_{k}} x_{n_{k}}(i) e_{i}+\sum_{i=i_{k}+1}^{\infty} x_{n_{k}}(i) e_{i} \\
& -\sum_{i=1}^{i_{j-1}} x_{n_{j}}(i) e_{i}-\sum_{i=i_{j-1}+1}^{i_{j}} x_{n_{j}}(i) e_{i}-\sum_{i=i_{j}+1}^{\infty} x_{n_{j}}(i) e_{i} \| \\
\geq & \left\|\sum_{i=i_{k-1}+1}^{i_{k}} x_{n_{k}}(i) e_{i}-\sum_{i=i_{j-1}+1}^{i_{j}} x_{n_{j}}(i) e_{i}\right\|-4 \delta_{0} .
\end{aligned}
$$


By

$$
\begin{aligned}
& I_{\Phi}\left(\sum_{i=i_{k-1}+1}^{i_{k}} x_{n_{k}}(i) e_{i}-\sum_{i=i_{j-1}+1}^{i_{j}} x_{n_{j}}(i) e_{i}\right) \\
& \quad=I_{\Phi}\left(\sum_{i=i_{k-1}+1}^{i_{k}} x_{n_{k}}(i) e_{i}\right)+I_{\Phi}\left(\sum_{i=i_{j-1}+1}^{i_{j}} x_{n_{j}}(i) e_{i}\right) \\
& \quad \geq \frac{1}{2}+\frac{\varepsilon_{0}}{3}+\frac{1}{2}+\frac{\varepsilon_{0}}{3}=1+\frac{2 \varepsilon_{0}}{3},
\end{aligned}
$$

we have

$$
\left\|\left(\sum_{i=i_{k-1}+1}^{i_{k}} x_{n_{k}}(i) e_{i}-\sum_{i=i_{j-1}+1}^{i_{j}} x_{n_{j}}(i) e_{i}\right)\right\| \geq 1+5 \delta_{1} \geq 1+5 \delta_{0} .
$$

Hence

$$
\left\|x_{n_{k}}-x_{n_{j}}\right\| \geq\left\|\sum_{i=i_{k-1}+1}^{i_{k}} x_{n_{k}}(i) e_{i}-\sum_{i=i_{j-1}+1}^{i_{j}} x_{n_{j}}(i) e_{i}\right\|-4 \delta_{0} \geq 1+\delta_{0} .
$$

This contradicts with the inequality $D\left[\left(x_{n}\right)\right] \leq 1$.

For convenience, we may assume that $I_{\Phi}\left(x_{n}\right) \leq \frac{1}{2}+\varepsilon$ for all $n \in N$. Hence

$$
I_{\Phi}\left(\frac{1-\varepsilon}{1+\varepsilon} x_{n}\right) \leq \frac{1-\varepsilon}{1+\varepsilon} I_{\Phi}\left(x_{n}\right) \leq \frac{1-\varepsilon}{1+\varepsilon}\left(\frac{1}{2}+\varepsilon\right)=\frac{1}{2}
$$

for all $n \in N$.

Let us take an element $x \in l_{\Phi}$ with $\|x\|=a$. By $\Phi \in \delta_{2}$, for any $\varepsilon>0$ there exists $i_{0}>0$ such that $\left\|\sum_{i=i_{0}+1}^{\infty} x(i) e_{i}\right\|<\varepsilon$. Put $x_{0}=a \frac{\sum_{i=i_{0}+1}^{\infty} x(i) e_{i}}{\left\|\sum_{i=i_{0}+1}^{\infty} x(i) e_{i}\right\|}$. Since $x_{n} \stackrel{w^{*}}{\rightarrow} 0$, there is a $n_{0} \in N$ such that $\left\|\sum_{i=1}^{i_{0}} x_{n}(i) e_{i}\right\|<\varepsilon$ when $n \geq n_{0}$.

Hence

$$
\begin{aligned}
\left\|x_{n}-x\right\| & \leq\left\|\sum_{i=1}^{i_{0}}\left(x_{n}(i)-x(i)\right) e_{i}+\sum_{i=i_{0}+1}^{\infty}\left(x_{n}(i)-x(i)\right) e_{i}\right\| \\
& \leq\left\|\sum_{i=1}^{i_{0}} x(i) e_{i}+\sum_{i=i_{0}+1}^{\infty} x_{n}(i) e_{i}\right\|+2 \varepsilon .
\end{aligned}
$$

We next estimate the $\left\|\sum_{i=1}^{i_{0}} x(i) e_{i}+\sum_{i=i_{0}+1}^{\infty} x_{n}(i) e_{i}\right\|$. Put $z_{n}=\frac{1-\varepsilon}{1+\varepsilon} \sum_{i=i_{0}+1}^{\infty} x_{n}(i) e_{i}$ for $n \geq n_{0}$. We have

$$
\begin{aligned}
& I_{\Phi}\left(\frac{a \frac{\sum_{i=1}^{i_{0}} x(i) e_{i}}{\left\|\sum_{i=1}^{i_{0}} x(i) e_{i}\right\|}-\frac{1-\varepsilon}{1+\varepsilon} \sum_{i=i_{0}+1}^{\infty} x_{n}(i) e_{i}}{d_{\Phi}+\varepsilon}\right) \\
& \quad=I_{\Phi}\left(\frac{x_{0}}{d_{\Phi}+\varepsilon}\right)+I_{\Phi}\left(\frac{z_{n}}{d_{\Phi}+\varepsilon}\right)
\end{aligned}
$$




$$
\begin{aligned}
& \leq I_{\Phi}\left(\frac{x_{0}}{c_{x_{0}, z_{n}}}\right)+I_{\Phi}\left(\frac{z_{n}}{c_{x_{0}, z_{n}}}\right) \\
& =1
\end{aligned}
$$

whence

$$
\left\|a \frac{\sum_{i=1}^{i_{0}} x(i) e_{i}}{\left\|\sum_{i=1}^{i_{0}} x(i) e_{i}\right\|}-\frac{1-\varepsilon}{1+\varepsilon} \sum_{i=i_{0}+1}^{\infty} x_{n}(i) e_{i}\right\| \leq d_{\Phi}+\varepsilon
$$

for $n \geq n_{0}$. Therefore, we obtain the inequalities

$$
\begin{aligned}
& \left\|\sum_{i=1}^{i_{0}} x(i) e_{i}-\sum_{i=i_{0}+1}^{\infty} x_{n}(i) e_{i}\right\| \\
& \quad \leq\left\|x_{0} \frac{\left\|\sum_{i=1}^{i_{0}} x(i) e_{i}\right\|}{a}-\frac{1-\varepsilon}{1+\varepsilon} \sum_{i=i_{0}+1}^{\infty} x_{n}(i) e_{i}-\frac{2 \varepsilon}{1+\varepsilon} \sum_{i=i_{0}+1}^{\infty} x_{n}(i) e_{i}\right\| \\
& \leq a\left(\frac{\left\|\sum_{i=1}^{i_{0}} x(i) e_{i}\right\|}{a}-1\right)+\left\|a \frac{\sum_{i=1}^{i_{0}} x(i) e_{i}}{\left\|\sum_{i=1}^{i_{0}} x(i) e_{i}\right\|}-z_{n}\right\|+\frac{2 \varepsilon}{1+\varepsilon}\left\|\sum_{i=i_{0}+1}^{\infty} x_{n}(i) e_{i}\right\| \\
& \quad \leq a\left(\frac{a+\varepsilon}{a}-1\right)+\left\|a \frac{\sum_{i=1}^{i_{0}} x(i) e_{i}}{\left\|\sum_{i=1}^{i_{0}} x(i) e_{i}\right\|}-z_{n}\right\|+\frac{2 \varepsilon}{1+\varepsilon} \\
& \quad \leq a \varepsilon+d_{\Phi}+\varepsilon+\frac{2 \varepsilon}{1+\varepsilon}=d_{\Phi}+\left(\frac{2+(1+a)(1+\varepsilon)}{1+\varepsilon}\right) \varepsilon .
\end{aligned}
$$

Therefore, we have $\left\|x_{n}-x_{0}\right\| \leq d_{\Phi}+\left(\frac{2+(1+a)(1+\varepsilon)}{1+\varepsilon}\right) \varepsilon+2 \varepsilon$. By the arbitrariness of $\varepsilon>0$, we get the inequality $R\left(\ell_{\Phi}\right) \leq d_{\Phi}$.

Summing up, we see that the equality $R\left(\ell_{\Phi}\right)=d_{\Phi}$ holds.

For any $x \in \ell_{\Phi}$ with $\|x\|=1$ and $N(x)=\{i \in N: x(i) \neq 0\}$ being finite we define $c_{x}$ as follows:

$$
\begin{aligned}
\tilde{c}_{x}= & \lim _{n \rightarrow \infty} \sup \left\{c_{x, y}>0: I_{\Phi}\left(\frac{x}{c_{x, y}}\right)+I_{\Phi}\left(\frac{y}{c_{x, y}}\right)=1 \text { for } y \in \ell_{\Phi} \text { with } I_{\Phi}(y) \leq 1,\right. \\
& n \leq N(y)<\infty\} .
\end{aligned}
$$

Corollary 2.4 If $\Phi \in \delta_{2}$ then $R\left(l_{\Phi}\right)=\sup \left\{\tilde{c}_{x}: x \in \ell_{\Phi}\right.$ with $\|x\|=1$ and $N(x)$ being finite $\}$.

Proof The proof is similar to the proof of Theorem 2.3.

Corollary 2.5 If $\Phi \in \delta_{2}$ and $\Phi \in \bar{\delta}_{2}$ then $R\left(l_{\Phi}, a\right)=R^{*}\left(l_{\Phi}, a\right)$ for any $0<a \leq 1$.

Theorem 2.6 $R^{*}\left(\ell_{\Phi}, 1\right)<2$ if and only if $\ell_{\Phi} \in \delta_{2}$ and $\Phi \in \bar{\delta}_{2}(1)$.

Proof Necessity. We only need to prove the necessity of $\Phi \in \bar{\delta}_{2}(1)$. Suppose that $\Phi \notin \bar{\delta}_{2}(1)$. Then for any natural number $k$ there exists $x_{k} \in S\left(\ell_{\Phi}\right)$ with $N\left(x_{k}\right) \geq k$ such that

$$
I_{\Phi}\left(\frac{x_{k}}{2}\right)>\frac{1-\frac{1}{k}}{2} I_{\Phi}\left(x_{k}\right) .
$$


For any fixed $k \in N$ and for any $\varepsilon>0$, there exists $i_{k} \in N$ such that $\sum_{i=k}^{i_{k}} \Phi_{i}\left(x_{k}(i)\right)>1-\varepsilon$. Put $\bar{x}_{k}=\frac{\sum_{i=k}^{i_{k}} x_{k}(i) e_{i}}{\left\|\sum_{i=k}^{i_{k}} x_{k}(i) e_{i}\right\|}$. Then

$$
\begin{aligned}
I_{\Phi}\left(\frac{\bar{x}_{k}}{2}\right)+I_{\Phi}\left(\frac{x_{i_{k}}}{2}\right) & =I_{\Phi}\left(\frac{\sum_{i=k}^{i_{k}} x_{k}(i) e_{i}}{2\left\|\sum_{i=k}^{i_{k}} x_{k}(i) e_{i}\right\|}\right)+I_{\Phi}\left(\frac{x_{i_{k}}}{2}\right) \\
& \geq I_{\Phi}\left(\frac{\sum_{i=k}^{i_{k}} x_{k}(i) e_{i}}{2}\right)+I_{\Phi}\left(\frac{x_{i_{k}}}{2}\right)+I_{\Phi}\left(\frac{\sum_{i=i_{k}+1}^{\infty} x_{k}(i) e_{i}}{2}\right)-\varepsilon \\
& =I_{\Phi}\left(\frac{x_{k}}{2}\right)+I_{\Phi}\left(\frac{x_{i_{k}}}{2}\right)-\varepsilon \geq \frac{1-\frac{1}{k}}{2}+\frac{1-\frac{1}{i_{k}}}{2}-\varepsilon \\
& =1-\frac{1}{k}-\varepsilon,
\end{aligned}
$$

which shows that $\left\|\bar{x}_{k}+x_{i_{k}}\right\| \geq 2\left(1-\frac{1}{k}-\varepsilon\right)$. Hence $c_{\bar{x}_{k}, x_{i}} \geq 2\left(1-\frac{1}{k}-\varepsilon\right)$. By the arbitrariness of $\varepsilon>0$ and $k \in N$, we see that $R\left(\ell_{\Phi}\right)=2$.

Sufficiency. Since $\Phi \in \bar{\delta}_{2}(1)$, there exist $\varepsilon_{0} \in(0,1)$ and $i_{\varepsilon} \in \mathcal{N}$ such that

$$
I_{\Phi}\left(\frac{x}{2}\right) \leq \frac{1-\varepsilon_{0}}{2} I_{\Phi}(x)
$$

whenever $I_{\Phi}(x)=1$ and $N(x) \geq i_{\varepsilon}$. For any $x \in S\left(\ell_{\Phi}\right)$ with finite $N(x)$ and $y \in S\left(\ell_{\Phi}\right)$ with $N(y) \geq n$, we may assume without loss of generality that $\max \{i: i \in N(x)\}<n$ and $n \geq i_{\varepsilon}$. Hence

$$
I_{\Phi}\left(\frac{x}{2}\right)+I_{\Phi}\left(\frac{y}{2}\right) \leq \frac{1}{2}+\frac{1-\varepsilon}{2}=\frac{2-\varepsilon}{2} .
$$

Since $\Phi \in \delta_{2}$, there exists $0<\alpha<1$ such that

$$
\|z\| \leq \alpha \quad \text { whenever } I_{\Phi}(z) \leq \frac{2-\varepsilon}{2}
$$

Therefore $\left\|\frac{x+y}{2}\right\| \leq \alpha$, i.e., $\|x+y\| \leq 2 \alpha$. Note that $c_{x, y}=\|x+y\|$. Hence $R\left(\ell_{\Phi}\right) \leq 2 \alpha<2$.

Theorem $2.7 R^{*}\left(\ell_{\Phi}, a\right)<1+a$ for $0<a<1$ if and only if $\Phi \in \delta_{2}$.

Proof We only need to prove the sufficiency. For any $0<\varepsilon<\frac{1}{2}$, by $\Phi \in \delta_{2}$, there exists a $d_{0}>0$ such that $\|x\| \leq 1-d$ whenever $I_{\Phi}(x) \leq \frac{1}{2}+\varepsilon$. Hence $\left\|x_{n}\right\| \leq 1-d$ if $n$ large enough for any weakly star null sequence $\left\{x_{n}\right\} \subset B\left(l_{\Phi}\right)$ with $D\left[\left(x_{n}\right)\right] \leq 1$. Hence

$$
\liminf _{n \rightarrow \infty}\left\|x_{n}-x\right\| \leq 1-d+a<1+a
$$

that is, $R^{*}\left(l_{\Phi}, a\right)<1+a$.

Example 2.8 Let $\Phi_{n}(u)=\left\{\begin{array}{ll}u^{2} & \text { if } u \leq \frac{1}{n}, \\ a_{n} u+b_{n} & \text { if } \frac{1}{n} \leq u \leq \infty,\end{array}\right.$, where $a_{n}=\frac{2}{n}, b_{n}=-\frac{1}{n^{2}}$. Then $\Phi_{n}$ is an Orlicz function for each $n \in N$.

$$
\text { If } u \leq \frac{1}{n} \text { and } 2 u>\frac{1}{n} \text {, then } \frac{1}{2 n}<u \leq \frac{1}{n} \text {. Hence }
$$

$$
\Phi_{n}(2 u)=\frac{2}{n} 2 u-\frac{1}{n^{2}} \leq \frac{4}{n^{2}}-\frac{1}{n^{2}}=\frac{3}{n^{2}} \leq 12 \frac{1}{4 n^{2}} \leq 12 u^{2}=12 \Phi_{n}(u) .
$$


If $0<u \leq \frac{1}{2 n}$ then $\Phi_{n}(2 u)=4 \Phi_{n}(u)$. If $\frac{1}{n}<u \leq 1$ then $\Phi_{n}(2 u) \leq 2 \Phi_{n}(u)$. If we put $K=24$ and $u_{0}=1$, then

$$
\Phi_{n}(2 u) \leq K \Phi_{n}(u) \text { for all } n \in N
$$

that is, $\Phi \in \delta_{2}$.

If $0<a<1$ then $R^{*}\left(l_{\Phi}, a\right)<1+a$.

Let us take $x_{n}=(0,0, \ldots, 0, \overbrace{\frac{n}{2}+\frac{1}{2 n}}^{n \text {th }}, 0, \ldots)$ for any $n \in N$. Then $I_{\Phi}\left(x_{n}\right)=1$ and

$$
I_{\Phi}\left(\frac{x_{n}}{2}\right)=\frac{2}{n}\left(\frac{n}{4}+\frac{1}{4 n}\right)-\frac{1}{n^{2}}=\frac{1}{2}-\frac{1}{2 n^{2}}
$$

whence $\lim _{n \rightarrow \infty} I_{\Phi}\left(\frac{x_{n}}{2}\right)=\frac{1}{2}$. Therefore $\Phi \notin \delta_{2}(1)$, which implies that $R^{*}\left(l_{\Phi}, 1\right)=2$.

Let $\left\{p_{k}\right\}_{k=1}^{\infty}$ be a sequence of real increasing numbers with $1<p_{1}$ and $\lim _{n \rightarrow \infty} p_{n}=p<\infty$. Then we have the following.

Theorem 2.9 Let $l^{\left(p_{i}\right)}$ be a Nakano sequence space equipped with the Luxemburg norm. Then $R\left(l^{\left(p_{i}\right)}\right)=2^{\frac{1}{p}}$ and $R\left(l_{\Phi}, a\right)=\left(\frac{1}{2}+a^{p}\right)^{\frac{1}{p}}, 0<a \leq 1$.

Proof Since $1<\inf \left\{p_{i}\right\} \leq \sup \left\{p_{i}\right\}=p<\infty$, the Nakano space equipped with the Luxemburg norm is reflexive. For any $x, y \in S\left(l^{\left(p_{i}\right)}\right)$ with $N(x), N(y)$ being finite. We now consider the following equation:

$$
I_{\Phi}\left(\frac{x}{c}\right)+I_{\Phi}\left(\frac{y}{c}\right)=1
$$

that is,

$$
\sum_{i=1}^{\infty}\left|\frac{x(i)}{c}\right|^{p_{i}}+\sum_{i=1}^{\infty}\left|\frac{y(i)}{c}\right|^{p_{i}}=1 .
$$

Then

$$
\frac{1}{c^{p}} \sum_{i=1}^{\infty}|x(i)|^{p_{i}}+\frac{1}{c^{p}} \sum_{i=1}^{\infty}\left|x_{n}(i)\right|^{p_{i}}=\frac{2}{c^{p}} \leq 1,
$$

i.e., $c \leq 2^{\frac{1}{p}}$. This shows the inequality $R\left(l^{\left(p_{i}\right)}, 1\right) \leq 2^{\frac{1}{p}}$. Take the classical basic sequence $\left\{e_{n}\right\} \subset S\left(l^{\left(p_{i}\right)}\right)$. If $c_{n, m}$ is a solution of the equation

$$
I_{\Phi}\left(\frac{e_{n}}{c}\right)+I_{\Phi}\left(\frac{e_{m}}{c}\right)=1
$$

and assuming without loss of generality that we may take $n>m$, we have the inequality

$$
\frac{2}{c^{p_{n}}} \geq 1
$$


Hence $R\left(l^{\left(p_{i}\right)}\right) \geq 2^{\frac{1}{p}}$. Together with the opposite inequality proved already, we have $R\left(l^{\left(p_{i}\right)}\right)=2^{\frac{1}{p}}$.

If $0<a<1$, for $x \in B\left(l^{\left(p_{i}\right)}\right)$ with finite $N(x)$ and $I_{\Phi}(x)=\frac{1}{2}$ and $y \in B\left(l^{\left(p_{i}\right)}\right)$ with finite $N(y)$ and $\|x\|=a$, we consider the following equation:

$$
I_{\Phi}\left(\frac{x}{c_{x, y}}\right)+I_{\Phi}\left(\frac{y}{c_{x, y}}\right)=1
$$

that is, the equation

$$
\sum_{i=1}^{\infty}\left|\frac{x(i)}{c_{x, y}}\right|^{p_{i}}+\sum_{i=1}^{\infty}\left|\frac{y(i)}{c_{x, y}}\right|^{p_{i}}=1
$$

Hence

$$
\frac{1}{c_{x, y}^{p}} \sum_{i=1}^{\infty}|x(i)|^{p_{i}}+\frac{a^{p_{\max }\{i: i \in N(y)\}}}{c_{x, y}^{p}} \sum_{i=1}^{\infty}\left|\frac{y(i)}{a}\right|^{p_{i}}=\frac{1}{2 c_{x, y}^{p}}+\frac{a^{p_{\max }\{i: i \in N(y)\}}}{c_{x, y}^{p}} \geq 1
$$

where $c_{x, y}^{p} \leq \frac{1}{2}+a^{p_{\max }\{i: i \in N(y)\}}$. Therefore $R\left(l_{\Phi}, a\right) \leq\left(\frac{1}{2}+a^{p}\right)^{\frac{1}{p}}$

Taking $x_{n}=\left(0, \ldots, 0,\left(\frac{1}{2}\right)^{\frac{1}{p_{n}}}, 0, \ldots\right)$ and $x_{m}=(0, \ldots, 0, \stackrel{m \text { th }}{a}, 0, \ldots)$, we get $I_{\Phi}\left(x_{n}\right)=\frac{1}{2}$ and $I_{\Phi}\left(\frac{x_{m}}{a}\right)=1$, which implies the equality $\left\|x_{m}\right\|=a$.

For any $n \neq m$, if $c>0$ is such that

$$
I_{\Phi}\left(\frac{x_{n}+x_{m}}{c}\right)=1
$$

then

$$
\left(\frac{1}{2}+a^{p_{m}}\right)^{\frac{1}{\max \left(p_{n}, p_{m}\right\}}} \leq c
$$

Letting $n, m \rightarrow \infty$, we get $R\left(l_{\Phi}, a\right) \leq\left(\frac{1}{2}+a^{p}\right)^{\frac{1}{p}}$, that is, $R\left(l_{\Phi}, a\right)=\left(\frac{1}{2}+a^{p}\right)^{\frac{1}{p}}$.

\section{Competing interests}

The author declares that they have no competing interests.

\section{Author details}

School of Mathematical Science, Harbin Normal University, Harbin, 150025, China.

\section{Acknowledgements}

The author would like to express sincere appreciation to the referees for their very helpful suggestions and kind comments. The author is supported by National Natural Science Foundation of China (Grant No. 11401143 and No. 11471091), the National Science Center of Poland (No. UMO-2012/07/B/ST1/03360), Overseas Returning Foundation of Hei Long Jiang Province (Grant No. LC201402) and Scientific Research Foundation of Heilongjiang Provincial Education Department (12541232).

Received: 3 September 2015 Accepted: 26 November 2015 Published online: 09 December 2015

\section{References}

1. Benavides, TD: A geometrical coefficient implying the fixed point property and stability results. Houst. J. Math. 22(4), 835-849 (1996)

2. Kirk, WA: A fixed point theorem for mappings which do not increase distances. Am. Math. Mon. 72, 1004-1006 (1965)

3. Prus, S: Nearly uniformly smooth Banach spaces. Boll. Unione Mat. Ital., B 3, 507-521 (1989)

4. Prus, S: Banach spaces with the uniform Opial property. Nonlinear Anal. 18, 697-704 (1992) 
5. García-Falset, J: Stability and fixed points for nonexpansive mapping. Houst. J. Math. 20(3), 495-505 (1994)

6. García-Falset, J: The fixed point property in Banach spaces with the NUS property. J. Math. Anal. Appl. 215(2), 532-542 (1997)

7. Goebel, K, Kirk, WA: Topics in Metric Fixed Point Theory. Cambridge University Press, Cambridge (1990)

8. Diestel, J: Sequence and Series in Banach Spaces. Graduate Texts in Mathematics. Springer, Berlin (1984)

9. Diestel, J: Geometry of Banach Spaces - Selected Topics. Lecture Notes in Mathematics, vol. 485. Springer, Berlin (1975)

10. Kantorovich, LV, Akilov, GP: Functional Analysis. Nauka, Moscow (1977) (in Russian)

11. Alherk, G, Hudzik, H: Uniformly non-I In Musielak-Orlicz spaces of Bochner type. Forum Math. 4(1), $403-410$ (1989)

12. Chen, ST: Geometry of Orlicz spaces. Diss. Math. 356, 1-204 (1996)

13. Musielak, J: Orlicz Spaces and Modular Spaces. Lecture Notes in Mathematics, vol. 1034. Springer, Berlin (1983)

14. Rao, MM, Ren, ZD: Theory of Orlicz Spaces. Dekker, New York (1991)

15. Chen, ST, Cui, YA, Hudzik, H, Sims, B: Geometric properties related to fixed point theory in some Banach function lattices. In: Kirk, WA, Sims, B (eds.) Handbook of Metric Fixed Point Theory, pp. 339-389. Kluwer Academic, Dordrecht (2001)

Submit your manuscript to a SpringerOpen ${ }^{\odot}$ journal and benefit from:

- Convenient online submission

Rigorous peer review

- Immediate publication on acceptance

- Open access: articles freely available online

- High visibility within the field

- Retaining the copyright to your article 